\title{
Engineering Applications of Distributed Fiber Optic Sensing Arrays
}

\author{
Dante Fratta \\ Geological Engineering \\ Civil and Environmental Engineering \\ University of Wisconsin-Madison
}

\begin{abstract}
Distributed Fiber Optic Sensing (DFOS) uses the interaction of laser pulses with fiber-optic imperfections to capture Brillouin, Rayleigh, and Raman scattering events. The interpretation of the scattering events with time-domain reflectometry captures temperatures, strains, and vibrations along the length of the arrays. These arrays can be up to tens of kilometers long and yield measurements at temporal sampling rates higher than $1 \mathrm{kHz}$, with spatial separation of less than $1 \mathrm{~m}$, sensing gauges from $2 \mathrm{~m}$ to $10 \mathrm{~m}$, temperature resolution as fine as $0.01{ }^{\circ} \mathrm{C}$, and dynamic strains in the order of less than microstrains. In this presentation, I review the physical principles governing the response of DFOS, engineering applications, and the challenges of interpreting DFOS data. Then, I discuss the use of DFOS arrays to monitor low-temperature geothermal fields' performance, measure strains in hard rock mines, sense surface and body waves in alluvial formations, and capture traffic noise signals. All these applications are inherent to the analysis of geological engineering systems and the monitoring of the civil infrastructure. Thus, the use of DFOS provides an opportunity to revolutionize how civil engineers interact, analyze, and evaluate data.
\end{abstract}

